\title{
La Logique Transactionnelle De L'appropriation Des Biens : Un Regard Sur La Structure Des Communes Camerounaises
}

(The Transactional Logic of the Ownership of Goods: A Look at the Structure of Cameroonian Municipalities)

\author{
BOOK NYOBE Aurélien Bertrand ${ }^{1}$, NDJECK Noé2 \\ ${ }^{1,2}$ Enseignant-Chercheur, Faculté des Sciences Economiques et de Gestion Appliquée/ Université De \\ Douala.
}

\begin{abstract}
Résumé
Dans la littérature économique, il a été démontré que les attributs de transactions impactent la structure des organisations. Cependant, les effets de l'appropriation de la terre et de la forêt communale sont moins connus. L'étude menée auprès de 18 communes, et appuyée par une analyse discrète des coûts de transaction montre que en fonction du degré de spécificité de l'actif, la gestion en régie et en régie intéressée impacteraient la structure financière. Celle-ci contribuerait à l'amélioration des recettes, à la prise en compte des externalités et du risque.
\end{abstract}

Mots clés : Appropriation des biens, structure de gouvernance, structure financière, coût de transactions, actifs spécifiques.

\begin{abstract}
:
In the economic literature, it has been shown that the attributes of transactions impact the structure of organizations. However, the effects of the appropriation of communal land and forest are less well known. The study carried out in $\mathbf{1 8}$ municipalities and supported by a discreet analysis of transaction costs, shows that depending on the degree of specificity of the asset, the regulated management and the management in interested party would impact the financial structure. This would help improve revenues, in taking into account externalities and risk.
\end{abstract}

Keywords: Ownership of goods, governance structure, financial structure, transaction costs, specific assets.

\section{Introduction}

L'appropriation des biens est depuis fort longtemps encrée dans les habitudes des communes des pays d'Afrique, d'une part par la volonté de leurs Etats de promouvoir la logique collective dans les Communes et d'autres part par les exigences des bailleurs de leur imposer l'initiative privée pour booster le développement local. Ainsi, l'appropriation des biens se fait à travers des transactions entre agents économiques avec comme support institutionnel les structures de gouvernances [Ghertman, 2003], dont la spécificité des actifs explique la structure financière [Williamson, 1988]. Au Cameroun, ces supports institutionnels sont encadrés par les articles 42, 46, 49 et 50 de la loi N²019/024 du 24 Décembre 2019 portant code général des collectivités territoriales décentralisés (CG_CTD) et appuyés par divers textes règlementaires pour promouvoir la gestion services locaux. Sur le plan pratique, les Communes disposent des parcelles de terres et de forets (articles 29, 30, 35 et 36(4) du code géneral des collectivités térritoriales décentralisées (CGCTD) sur la gestion et l'utilisation d'une partie du domaine privé, public et national de l'Etat au profit des Collectivités Territoriales). La gestion et l'exploitation de ces biens est marquée par des transactions dans les Communes et des Communes vers les partenaires privés ou des regroupements communaux qui ont désormais les droits d'usage, de jouissance et de disposer desdits biens. Théoriquement deux logiques s'affrontent sur les différentes transactions liées à l'appropriation des terres et des forêts propriétés de la Commune. La première logique dite collective ou commune met en avant les vertus de la 
décentralisation considérée comme un moyen de donner aux populations locales plus de droit sur les ressources naturelles [Anderson et al, 2006 ; Agrawal et Ostrom, 2001 ; Agrawal et Ribot, 1999 ; Ribot, 2003]. Cette logique s'oppose à la logique dite d'accaparement ou privée qui considère l'appropriation des biens pour un usage économique et à des fins lucratif, [Maurel, 1994, 2012 ; Simard, 2007, p.11 ; BissuelRoy, 2010]. Confrontées à de nombreuses exigences en matière de financement de leurs plans communaux de développement, les Communes des Etats Africains, notamment celles du Cameroun doivent satisfaire à cette exigence. Dans cette perspective, Quelle logique d'appropriation des biens doit influencer la structure des Communes ? Quels sont les attributs de transactions qui vont influencer les modes de gouvernances dans les Communes? Quels sont les modes gouvernance les plus efficaces pour l'appropriation des biens? Quels sont les modes de financement les plus efficients adaptés a chaque structure de gouvernance ? L'étude à pour objectif de comprendre l'effet de l'appropriation des biens sur les structures de gouvernance et financière des Communes Camerounaises. La gestion des transactions des biens et les apports desdits transactions sur le développement local sont analysés.

\section{La gestion transactionnelle des biens}

Mise en place pour une meilleure transformation des territoires locaux, elle est axée sur des transactions qui sont généralement soient collectives (1.1) soient privées (1.2).

\subsection{La Décentralisation comme logique collective ou de logique de mise en commun}

La décentralisation est un moyen d'améliorer la performance dans la réduction de la pauvreté et que ses bénéfices induisent l'amélioration de l'efficacité des services publics offerts, elle doit permettre de distribuer les services plus appropriés, une meilleure gouvernance et l'habilitation des citoyens locaux [Blair, 2000]. Cette définition met en exergue la promotion du développement local et la gouvernance territoriale. Le développement local est un processus qui vise à construire un mieux-être des populations à l'intérieur d'un espace donné avec une approche où les différents acteurs se rencontrent, échangent et édifient les projets de société ». Le développement local participe donc à la logique collective, car Il est fondé sur deux aspects le capital social (ensemble de normes et de réseaux qui facilitent l'action collective) et la proximité (due au fait qu'ils appartiennent au même réseau (logique d'appartenance) ou qu'ils partagent des valeurs et des objectifs communs (logique de similitude)). La mise en commun de la gouvernance territoriale signifie à la fois la participation des populations aux décisions par l'intermédiaire des différents groupes ou représentants, ainsi qu'une imbrication des niveaux de décision, du local vers le global. Elle responsabilise les acteurs locaux et par conséquent la participation citoyenne au niveau local. Dans cette perspective, la participation des acteurs est devenue une dimension essentielle de la mise en œuvre du concept de gestion durable des ressources naturelles. Plusieurs études ont souligné les vertus de la décentralisation considérée comme un moyen de donner aux populations locales plus de droit sur les ressources naturelles [Anderson et al, 2006], un processus de redistribution du pouvoir, des ressources et des facilités administratives à divers paliers de gouvernements et des communautés [Agrawal et Ostrom, 2001 ; Agrawal et Ribot, 1999], un processus essentiel pour la réduction de la pauvreté [Steiner, 2007] ou comme une reforme visant à améliorer la participation des populations pour une gouvernance locale efficace et équitable [Ribot, 2003]. À la faveur de la décentralisation de la gestion forestière au Cameroun, plusieurs opportunités ont été créées par la loi - forestière de 1994 pour encourager la participation des acteurs à la gestion forestière autant à l'échelle nationale (commission/comité ministériel ou interministériel) que locale (comités locaux de gestion forestière). Cette loi s'est traduite par la dévolution aux acteurs locaux la gestion des forêts communautaires et des redevances forestières [Jérémie, 2009]. Toutefois, la décentralisation apparait comme une convention d'effort dans la mesure où «elle constitue un vaste système métrologique destiné à fournir aux acteurs des repères quantitatifs conventionnels » [Gomez, 1996, p. 249]. De tels cadres permettent d'établir l'accord conventionnel sur l'effort selon un objectif d'efficience commun. Une des raisons qui réside dans la représentation partagée des efforts à fournir est basée sur la dimension du développement local. La transaction des biens, propriété de la commune tient compte de la participation et des aspirations des acteurs locaux qui intègrent de manière volontaire les externalités économiques, sociales, culturelles, environnementales de leurs activités de manière construire un mieux être collectif induisant la distribution équitable des services publics offerts appropriés. Par conséquent, ce n'est pas parce que les communes adoptent des conventions qu'elles ne disposent d'aucune liberté. En effet, les communes «peuvent tricher, désobéir, tenter de changer le système conventionnel en place, faire référence à des systèmes conventionnels 
différents... elles peuvent agir contre la convention » [Gomez, 1996, p. 177]. Ce type de comportement résulte d'une des conditions conventionnelles qui stipule qu'il existe au moins une autre proposition alternative à la résolution du problème d'appropriation des biens. En d'autres termes, différentes conventions pouvant se concurrencer pour le bien être collectif des populations locales. La commune choisit celle à laquelle elle souhaite adhérer.

\subsection{Esprit entrepreneurial comme logique d'accaparement ou logique privée}

C'est par l'Europe centrale que le collectivisme agraire a opéré une rupture irréversible qui s'exprime par la disparition du système de valeurs paysan et notamment du sentiment d'attachement à la terre, de tout esprit d'entreprise, mais aussi par le rôle de l'ancienne élite agricole qui aurait trouvé la voie d'une reconversion réussie [Maurel, 1994]. Ainsi, divers stratégies d'appropriation fondées sur la terre et le capital d'exploitation ont été instaurées. Entre autre, la restauration de pleins droits de propriété s'appliquant aux propriétaires dont les droits avaient étés limités lors de l'entrée en vigueur de la collectivisation agraire, les procédures de restitution ou de compensation s'appliquant précisément aux biens qui ont été expropriés où confisqués de manière illégale, enfin l'attribution des terres à certaines catégories d'ayants droit a constitué une autre forme de redistribution foncière. Ces stratégies d'appropriation ont permis aux ayants droit de réaliser une plus value économique. Toutefois, selon [Ripoll et Veschambre, 2005, p.6] «penser en terme [s] d'appropriation de l'espace conduit [...] à envisager l'occupation ou l'usage de l'espace, mais aussi sa production et son détournement, son marquage, sa valorisation ou inversement sa stigmatisation ». Les auteurs associent à leur définition les dimensions matérielle, idéelle et institutionnelle de l'appropriation de l'espace dans l'optique de comprendre la finalité des usages des terres agricoles et des forêts à travers l'embourgeoisement rural et la requalification des terres. L'embourgeoisement rural selon [Simard, 2007, p. 201] est un phénomène à la fois physique, économique, social et culturel, ce concept évoque une transformation des statuts d'occupation et une recomposition des propriétaires fonciers dans l'espace rural. Il implique le double processus de migration et de colonisation des classes moyenne et supérieure dans cet espace et le déplacement des classes plus modestes vers des lieux plus accessibles à l'extérieur. Les études sur la gentrification inspireront deux écoles de pensée, l'une mettant l'accent sur la production et les structures de l'organisation économique du phénomène, l'autre sur la consommation, la culture et le goût (life style) des « gentrifieurs » [Guimond et Simard, 2005 ; Perrenound, 2008]. Pour [Bissuel-Roy, 2010, p.21], la requalification est un processus qui implique la transformation « d'un lieu déjà construit inséré dans un milieu de vie ». Celle-ci s'applique à la fois aux aspects humains (par exemple, économique, social et culturel) et physiques (par exemple, l'aménagement, le zonage agricole) du territoire. En effet, en achetant une terre agricole, l'exploitant contribue à son réaménagement (changer l'orientation), à sa réhabilitation (rendre utilisable), à sa réaffectation (changer la vocation), à sa revalorisation (l'augmentation de sa valeur), à sa requalification (augmenter sa qualité) et à sa restauration (rénover selon le caractère), [Giasson et Provost, 2000, p. 189]. Toutes ces dimensions sont pertinentes afin de comprendre les requalifications à la fois physiques et organisationnelles des terres et les conséquences transactionnelles que cela peut avoir sur la structure financière de la Commune, bénéficiaire résiduel desdits transformations. Au Cameroun, les articles 42, 46, 49 et 50 de la loi N²019/024 du 24 Décembre 2019 portant code général des collectivités territoriales décentralisés ont mis en place divers modalités de gestion services locaux des Collectivités Territoriales décentralisées, notamment la gestion en régie et la gestion déléguée des services locaux dans le but de faciliter les transactions liées aux biens et services communaux (l'exploitation de terre au profit d'une élite agricole, et de la forêt communale).

\subsection{La structure analysée selon la logique transactionnelle}

L'analyse s'attardera sur les choix du mode de gouvernance et du mode de financement lors des transactions des biens entre agents économique dans les communes.

\subsubsection{Du choix de la structure de gouvernance}

Selon [Williamson, 1985] les transactions (échange qui implique au moins deux agents, plusieurs firmes ou unités opérationnelles au sein de la même organisation) entre agents économiques peuvent utiliser comme support institutionnel les modes de gouvernance. Williamson distingue trois formes de gouvernance discrètes. Le marché au sens de l'économie néo-classique c'est-à-dire un système de prix dans lequel la firme est une fonction de production. Les formes hybrides pour les contrats de fourniture ou de vente à court 
terme, contrat récurrent à plus long terme, accord de licence de fabrication de sous-traitance de franchise ou de marque, les alliances. La hiérarchie étudiée dans le cadre de la théorie des coûts de transactions est un lieu d'exercice du «fiat» c'est-à-dire l'acte d'autorité ou ordre qui tient lieu de mécanisme interne de coordination pour Williamson. [Williamson 1975, 1985] utilise la hiérarchie pour résoudre les problèmes de spécificité des actifs et d'opportunisme et [Barzel, 1982] pour réduire les coûts de mesure et de contrôle de l'activité économique. Les contrats représentent en effet l'étendue du champ d'application des décisions des agents économiques. Le type de contrat est donc suffisant pour mesurer les frontières de la firme. Certes la théorie des coûts de transaction permet de répondre à la question du plus ou moins de l'intégration verticale, mais la plus part des travaux empirique relative à cette théorie porte sur les attributs de transaction en tant que déterminants et éléments essentiels pour l'arbitrage entre les modes de gouvernance. Plusieurs attributs de transactions ont été convoqués. En premier lieu les hypothèses comportementales des individus à savoir la rationalité limité developpé par [Simon, 1945] et l'opportunisme apporté par [Alchian et Demsetz, 1972] et reprise par [Williamson, 1975]. Ensuite les hypothèses comportementales sur les transactions, la spécificité des actifs mis an avant par [Masten, 1996], l'incertitude [Delmas, 1996] et la fréquence. Enfin, les instruments de management que sont l'intensité des incitations, l'importance de la bureaucratie [Williamson, 1991] et le niveau de compétence que nous allons associer dans le cadre de notre étude. Ainsi, lorsque l'incertitude interne est élevée ou bien lorsque l'entreprise possède des compétences fortes, la firme choisit plutôt l'intégration verticale [Delmas, Ghertman et Heiman, 1997]. Les attributs de transactions évoqués plus haut influencent fortement les transactions inhérentes à l'appropriation foncière et forestière, biens des collectivités locales. Le droit d'usage, de jouissance et de disposer de la terre ou d'un titre forestier dépend des transactions entre la Commune et d'autres agents économiques (entreprises, particuliers...) et par conséquent des coûts de transaction, donc les transformations (biens) auront un impact sur les coûts de transaction. La théorie suggère qu'on choisisse le mode de gouvernance qui minimise le plus de coûts de transactions.

\subsubsection{Du choix de la structure financière}

Les travaux de [Cornell et Shapiro, 1987] ont souligné l'existence d'une relation directe entre la nature des transactions effectuées et sa structure financière, car celles-ci engendre des coûts de contrats implicites et explicites qui affecte la valeur de la firme. En outre, ils ajoutent qu'une structure financière peu endettée est une garantie pour les contractants de l'exécution d'un service dans les bonnes conditions. Par la suite [Williamson, 1988] applique les concepts de la théorie des coûts de transactions aux décisions de financement. Williamson considère que les fonds propres et l'endettement sont des structures de direction de la firme et nom pas des simples instruments financiers concurrents ou alternatifs. Il affirme que la solution du financement optimale est celle qui minimise les coûts de transaction entre les agents. Ces coûts sont largement influencés par la spécificité des actifs à financer. Il en découle que le choix d'une source de financement est déterminée par la spécificité des actifs à acquérir. Cela implique que le financement d'une entreprise reflètera la nature de la politique d'investissement. Williamson appréhende la structure financière comme une structure de régulation des coûts de transactions générées par la nature de l'investissement luimême dépendant du stade de vie de l'entreprise et surtout du degré de spécificité des actifs (Définit par son aptitude à être interchangeable ou transférable par sa facilité a être utilisé dans d'autres fonctions ou par d'autres utilisateurs) a financer. La théorie suggère que la dette sera utilisée pour des projets avec un bas niveau de spécificité d'actif, le financement par fonds propres sera utilisé quand le degré de spécificité de l'actif est élevé tandis que les formes hybrides de financement prévues pour les niveaux de spécificités intermédiaires. La réalisation d'investissement spécifique étant peu fréquente, l'endettement apparait comme l'instrument financier privilégié par les firmes préférées à l'augmentation du capital. En effet, [Williamson, 1988] confirme l'ordre de financement proposé par [Myers et Majluf, 1984] et ajoute que cet ordre est inversé lorsqu'il s'agit de financer un actif spécifique. La théorie des coûts de transactions explique les choix de financement non pas par la recherche d'un ratio d'endettement optimal comme le prédisent les théories d'agence et de compromis, mais par la spécificité de l'actif à financer. D'autres auteurs ont montré la relation entre la spécificité de l'actif et le financement. [Rajan et Zingales, 1995] montrent qu'il existe une relation positive entre la tangibilité des actifs d'une firme et son endettement mesuré en valeur comptable et marchande. [Abimbola, 2002] obtient le même résultat en montrant que l'endettement est positivement corrélé à la liquidation des actifs. [Vilasuo et Minkler, 2001] trouvent que le financement par fonds propres permet de réduire les coûts des transactions quand les actifs à financer sont très spécifiques et offre une 
meilleure protection aux obligataires contre le risque de défaillance et réduit ainsi les coûts d'agence de la dette. [Cheng et Shiu, 2007] observent les décisions de financements d'en échantillon d'entreprise de 45 pays et trouvent que les firmes dont les actifs sont très tangibles ont les ratios d'endettement à long terme les plus élevés. [Chakraborty, 2010] obtient le même résultat. Cependant l'étude de [Booth et Al, 2001] qui a porté sur dix pays en voie de développement montre qu'il existe une relation négative entre la tangibilité des actifs et le niveau d'endettement. [Huang et Song, 2006)] obtiennent le même résultat et montre que l'endettement des entreprises chinoises est négativement corrélé avec le degré de tangibilité des actifs. La spécificité de l'actif est le plus souvent agrégéé par l'incertitude et la fréquence de la transaction, puisqu'elle est leur déterminant. Compte tenu de ce qui précède deux propositions théoriques sont formulées

P1 : Les attributs de transactions de l'appropriation des biens de la commune impactent le choix du mode de gouvernance dans cette commune

P2 : La spécificité des actifs de l'appropriation des biens de la commune impactent le choix du mode de financement dans cette commune

\section{Approche méthodologique}

Notre étude questionne l'impact de l'appropriation des biens sur la structure financière des Communes et sa transformation sur leur développement local. Pour répondre à cette question, nous allons présenter d'abord la méthodologie et les résultats obtenus (2.1.) ensuite les principaux apports de l'étude (2.2).

\subsection{Méthodologie et résultats obtenus}

L'analyse de notre méthodologie permet de s'appesantir sur la méthode de collecte et d'analyse des données (2.1.1), l'analyse synthétique des résultats (2.1.2) et la discussion des résultats (2.1.3).

\subsubsection{Méthode de collecte et d'analyse des données}

L'analyse de notre étude est essentiellement basée sur 18 cas [Yin, 1994, P.13]. Les données collectées concernent les transactions issues de l'appropriation des biens dans les collectivités pour la période (2016 à 2020). Un guide d'entretien à été préalablement envoyé aux Communes et aux partenaires privés et expliqué avant chaque entrevue. L'entrevue était organisée avec les cadres municipaux des communes, les responsables financiers des entreprises agricoles et entreprises forestières dans la région du littoral et de l'est du Cameroun. Ces entreprises exploitent les produits agricoles (palmier à huile, banane plantain) et les produits forestiers ligneux (bois). Le traitement des données à été facilité par la méthode de l'analyse discrète des modes de gouvernance proposé par [Williamson, 1991]. Cette méthode permet de voir quel est le mode de gouvernance qui économise le plus les coûts de transactions et par ricochet le mode de financement le plus efficient. Concrètement les coûts de transaction ne sont pas calculés, mais une estimation relative de chaque coût de transaction par mode de gouvernance et par attribut faite. Pour traiter les données recueillies au cours des entretiens exploratoires, nous avons choisis de procéder par une analyse de contenu dont l'objet est de faire émerger des questionnements nouveaux permettant de compléter les lectures théoriques. Elle a été retenue, car elle vise la manipulation de messages (contenu et expression de ce contenu) pour mettre à jour des indicateurs permettant d'inférer sur une réalité autre que celle du message [Bardin, 1998]. Le guide d'entretien utilisé comprend outre les questions d'ordre général, les questions sur les attributs liés à l'appropriation des biens (terres et titre de forêt), les modes de gouvernances et les modes de financement. Les attributs de transactions liés à l'appropriation des biens sont appréciés par l'opportunisme (OPPOR), la spécificité des actifs (SPACT), l'incertitude (INCER), la fréquence (FREQU), l'incitation (INCIT), la bureaucratie (BUREA) et les compétences (COMPE) révélées susceptible de transformer la structure financière. Parmi les variables caractéristiques des modes de gouvernance nous avions retenus les modes de gestion des collectivités territoriales issus des articles 42, 46, 49 et 50 du CGCTD de la loi 2013/024 du 24 décembre 2019 portant code général des collectivités territoriales décentralisées sur les modalités de gestion des services locaux des Collectivités Territoriales notamment la gestion en régie (GESRE), la gestion en régie intéressée (REINT), le groupement des collectivités (SYNCO). Les modes de financement retenus sont les recettes des Collectivités Territoriales issus des articles 390, 392, 393, 398 et 399 du CGCTD les fonds propres (FPR) constitués des impôts locaux, du produit de l'exploitation des domaines, des dotations des subventions et des autres recettes), les dettes (DET) qui comprennent les emprunts, enfin le mode hybride c'est-à-dire dettes et fonds propres (DFP). Pour ce qui est de la spécificité des actifs nous avons la spécificité des actifs bas (SPA), la spécificité des actifs 
intermédiaire (SPI) et la spécificité des actifs élevés (SPE).

\subsubsection{Analyse synthétique des résultats}

Tableau 1. Transactions liées à l'appropriation de la terre

\begin{tabular}{|c|c|c|c|c|c|c|c|c|c|}
\hline & & \multicolumn{3}{|c|}{ MODES DE GOUVERNANCE } & & & \multicolumn{3}{|c|}{ MODES DE GOUVERNANCE } \\
\hline & ATTRIBUTS & REINT & SYNCO & GESRE & & ATTRIBUTS & REINT & SYNCO & GESRE \\
\hline \multirow{9}{*}{ Cas 1} & OPPOR & 1 & 2 & 3 & \multirow{7}{*}{ Cas 2} & OPPOR & 1 & 2 & 3 \\
\hline & SPACT & 3 & 2 & 1 & & SPACT & 3 & 2 & 1 \\
\hline & INCER & 3 & 2 & 1 & & INCER & 3 & 2 & 1 \\
\hline & FREQU & 3 & 2 & 1 & & FREQU & 3 & 2 & 1 \\
\hline & INCIT & 3 & 2 & 1 & & INCIT & 3 & 2 & 1 \\
\hline & BUREA & 1 & 2 & 3 & & BUREA & 1 & 2 & 3 \\
\hline & COMPE & 1 & 2 & 3 & & COMPE & 1 & 2 & 3 \\
\hline & & \multicolumn{3}{|c|}{ MODES DE GOUVERNANCE } & & & \multicolumn{3}{|c|}{ MODES DE GOUVERNANCE } \\
\hline & ATTRIBUTS & REINT & SYNCO & GESRE & & ATTRIBUTS & REINT & SYNCO & GESRE \\
\hline \multirow{9}{*}{ Cas 3} & OPPOR & 1 & 2 & 3 & \multirow{7}{*}{ Cas 4} & OPPOR & 1 & 2 & 3 \\
\hline & SPACT & 3 & 2 & 1 & & SPACT & 1 & 2 & 3 \\
\hline & INCER & 3 & 2 & 1 & & INCER & 3 & 2 & 1 \\
\hline & FREQU & 3 & 2 & 1 & & FREQU & 3 & 2 & 1 \\
\hline & INCIT & 3 & 2 & 1 & & INCIT & 3 & 2 & 1 \\
\hline & BUREA & 1 & 2 & 3 & & BUREA & 1 & 2 & 3 \\
\hline & COMPE & 1 & 2 & 3 & & COMPE & 1 & 2 & 3 \\
\hline & & \multicolumn{3}{|c|}{ MODES DE GOUVERNANCE } & & & \multicolumn{3}{|c|}{ MODES DE GOUVERNANCE } \\
\hline & ATTRIBUTS & REINT & SYNCO & GESRE & & ATTRIBUTS & REINT & SYNCO & GESRE \\
\hline \multirow{9}{*}{ Cas 5} & OPPOR & 1 & 2 & 3 & \multirow{7}{*}{ Cas 6} & OPPOR & 1 & 2 & 3 \\
\hline & SPACT & 3 & 2 & 1 & & SPACT & 3 & 2 & 1 \\
\hline & INCER & 3 & 2 & 1 & & INCER & 3 & 2 & 1 \\
\hline & FREQU & 3 & 2 & 1 & & FREQU & 3 & 2 & 1 \\
\hline & INCIT & 3 & 2 & 1 & & INCIT & 3 & 2 & 1 \\
\hline & BUREA & 1 & 2 & 3 & & BUREA & 1 & 2 & 3 \\
\hline & COMPE & 1 & 2 & 3 & & COMPE & 1 & 2 & 3 \\
\hline & & \multicolumn{3}{|c|}{ MODES DE GOUVERNANCE } & & & \multicolumn{3}{|c|}{ MODES DE GOUVERNANCE } \\
\hline & ATTRIBUTS & REINT & SYNCO & GESRE & & ATTRIBUTS & REINT & SYNCO & GESRE \\
\hline \multirow{9}{*}{ Cas 7} & OPPOR & 1 & 2 & 3 & \multirow{7}{*}{ Cas 8} & OPPOR & 1 & 2 & 3 \\
\hline & SPACT & 3 & 2 & 3 & & SPACT & 3 & 2 & 1 \\
\hline & INCER & 3 & 2 & 1 & & INCER & 3 & 2 & 1 \\
\hline & FREQU & 3 & 2 & 1 & & FREQU & 3 & 2 & 1 \\
\hline & INCIT & 3 & 2 & 1 & & INCIT & 3 & 2 & 1 \\
\hline & BUREA & 1 & 2 & 3 & & BUREA & 1 & 2 & 3 \\
\hline & COMPE & 1 & 2 & 3 & & COMPE & 1 & 2 & 3 \\
\hline & & \multicolumn{3}{|c|}{ MODES DE GOUVERNANCE } & & & \multicolumn{3}{|c|}{ MODES DE GOUVERNANCE } \\
\hline & ATTRIBUTS & REINT & SYNCO & GESRE & & ATTRIBUTS & REINT & SYNCO & GESRE \\
\hline \multirow{7}{*}{ Cas 9} & OPPOR & 1 & 2 & 3 & \multirow{7}{*}{ Cas 10} & OPPOR & 1 & 2 & 3 \\
\hline & SPACT & 3 & 2 & 3 & & SPACT & 3 & 2 & 1 \\
\hline & INCER & 3 & 2 & 1 & & INCER & 3 & 2 & 1 \\
\hline & FREQU & 3 & 2 & 1 & & FREQU & 3 & 2 & 1 \\
\hline & INCIT & 3 & 2 & 1 & & INCIT & 3 & 2 & 1 \\
\hline & BUREA & 1 & 2 & 3 & & BUREA & 1 & 2 & 3 \\
\hline & COMPE & 1 & 2 & 3 & & COMPE & 1 & 2 & 3 \\
\hline
\end{tabular}

1 : coût de transaction faible ; 2 : coût de transaction intermédiaire ; 3 : coût de transaction élevé

Tableau 2. Transactions liées l'appropriation de la forêt communale

\begin{tabular}{|c|c|c|c|c|c|c|c|c|c|}
\hline & & \multicolumn{3}{|c|}{ MODES DE GOUVERNANCE } & & & \multicolumn{3}{|c|}{ MODES DE GOUVERNANCE } \\
\hline & ATTRIBUTS & REINT & SYNCO & GESRE & & ATTRIBUTS & REINT & SYNCO & GESRE \\
\hline \multirow{9}{*}{ Cas 11} & OPPOR & 1 & 2 & 3 & \multirow{7}{*}{ Cas 12} & OPPOR & 1 & 2 & 3 \\
\hline & SPACT & 1 & 2 & 3 & & SPACT & 1 & 2 & 3 \\
\hline & INCER & 3 & 2 & 1 & & INCER & 3 & 2 & 1 \\
\hline & FREQU & 3 & 2 & 1 & & FREQU & 3 & 2 & 1 \\
\hline & INCIT & 3 & 2 & 1 & & INCIT & 3 & 2 & 1 \\
\hline & BUREA & 1 & 2 & 3 & & BUREA & 1 & 2 & 3 \\
\hline & COMPE & 1 & 2 & 3 & & COMPE & 1 & 2 & 3 \\
\hline & & \multicolumn{3}{|c|}{ MODES DE GOUVERNANCE } & & & \multicolumn{3}{|c|}{ MODES DE GOUVERNANCE } \\
\hline & ATTRIBUTS & REINT & SYNCO & GESRE & & ATTRIBUTS & REINT & SYNCO & GESRE \\
\hline \multirow{3}{*}{ Cas 13} & OPPOR & 1 & 2 & 3 & \multirow{3}{*}{ Cas 14} & OPPOR & 1 & 2 & 3 \\
\hline & SPACT & 3 & 2 & 1 & & SPACT & 1 & 2 & 3 \\
\hline & INCER & 3 & 2 & 1 & & INCER & 3 & 2 & 1 \\
\hline
\end{tabular}




\begin{tabular}{|c|c|c|c|c|c|c|c|c|c|}
\hline & FREQU & 3 & 2 & 1 & & FREQU & 3 & 2 & 1 \\
\hline & INCIT & 3 & 2 & 1 & & INCIT & 3 & 2 & 1 \\
\hline & BUREA & 1 & 2 & 3 & & BUREA & 1 & 2 & 3 \\
\hline & COMPE & 1 & 2 & 3 & & COMPE & 1 & 2 & 3 \\
\hline & & \multicolumn{3}{|c|}{ MODES DE GOUVERNANCE } & & & \multicolumn{3}{|c|}{ MODES DE GOUVERNANCE } \\
\hline & ATTRIBUTS & REINT & SYNCO & GESRE & & ATTRIBUTS & REINT & SYNCO & GESRE \\
\hline \multirow{9}{*}{ Cas 15} & OPPOR & 1 & 2 & 3 & \multirow{7}{*}{ Cas 16} & OPPOR & 1 & 2 & 3 \\
\hline & SPACT & 1 & 2 & 3 & & SPACT & 3 & 1 & 2 \\
\hline & INCER & 3 & 2 & 1 & & INCER & 3 & 2 & 1 \\
\hline & FREQU & 3 & 2 & 1 & & FREQU & 3 & 2 & 1 \\
\hline & INCIT & 3 & 2 & 1 & & INCIT & 3 & 2 & 1 \\
\hline & BUREA & 1 & 2 & 3 & & BUREA & 1 & 2 & 3 \\
\hline & COMPE & 1 & 2 & 3 & & COMPE & 1 & 2 & 3 \\
\hline & & \multicolumn{3}{|c|}{ MODES DE GOUVERNANCE } & & & \multicolumn{3}{|c|}{ MODES DE GOUVERNANCE } \\
\hline & ATTRIBUTS & REINT & SYNCO & GESRE & & ATTRIBUTS & REINT & SYNCO & GESRE \\
\hline \multirow{7}{*}{ Cas 17} & OPPOR & 1 & 2 & 3 & \multirow{7}{*}{ Cas 18} & OPPOR & 1 & 2 & 3 \\
\hline & SPACT & 2 & 1 & 3 & & SPACT & 1 & 2 & 3 \\
\hline & INCER & 3 & 2 & 1 & & INCER & 3 & 2 & 1 \\
\hline & FREQU & 3 & 2 & 1 & & FREQU & 3 & 2 & 1 \\
\hline & INCIT & 3 & 2 & 1 & & INCIT & 3 & 2 & 1 \\
\hline & BUREA & 1 & 2 & 3 & & BUREA & 1 & 2 & 3 \\
\hline & COMPE & 1 & 2 & 3 & & COMPE & 1 & 2 & 3 \\
\hline
\end{tabular}

1 : coût de transaction faible ; 2 : coût de transaction intermédiaire ; 3 : coût de transaction élevé

Tableau 3. Analyse synthétique globale de l'appropriation de la terre

\begin{tabular}{|c|c|c|c|c|c|c|c|c|c|c|c|c|}
\hline \multicolumn{13}{|c|}{ Analyse synthétique globale de l'appropriation de la terre } \\
\hline \multirow{3}{*}{ CAS } & \multicolumn{6}{|c|}{ PROPOSITION 1A } & \multicolumn{6}{|c|}{ PROPOSITION 2A } \\
\hline & \multicolumn{3}{|c|}{$\begin{array}{l}\text { COÛTS DE } \\
\text { TRANSACTIONS }\end{array}$} & \multicolumn{3}{|c|}{$\begin{array}{l}\text { MODES DE } \\
\text { GOUVERNANCE }\end{array}$} & \multicolumn{3}{|c|}{$\begin{array}{l}\text { SPECIFICITE } \\
\text { ACTIF }\end{array}$} & \multicolumn{3}{|c|}{$\begin{array}{l}\text { MODES DE } \\
\text { FINANCEMENT }\end{array}$} \\
\hline & CTB & CTI & CTE & REINT & SYNCO & GESRE & SAB & SAI & SAE & DET & DFP & FPE \\
\hline Cas 1 & 1 & 0 & 0 & 0 & 0 & 1 & 1 & 0 & 0 & 0 & 0 & 1 \\
\hline Cas 2 & 1 & 0 & 0 & 0 & 1 & 0 & 0 & 1 & 0 & 0 & 1 & 0 \\
\hline Cas 3 & 1 & 0 & 0 & 0 & 0 & 1 & 1 & 0 & 0 & 0 & 0 & 1 \\
\hline Cas 4 & 0 & 0 & 1 & 1 & 0 & 1 & 1 & 0 & 0 & 0 & 0 & 1 \\
\hline Cas 5 & 1 & 0 & 0 & 0 & 0 & 1 & 1 & 0 & 0 & 1 & 0 & 0 \\
\hline Cas 6 & 1 & 0 & 0 & 0 & 0 & 1 & 1 & 0 & 0 & 0 & 0 & 1 \\
\hline Cas 7 & 0 & 1 & 0 & 0 & 1 & 0 & 0 & 1 & 0 & 0 & 1 & 0 \\
\hline Cas 8 & 1 & 0 & 0 & 0 & 0 & 1 & 1 & 0 & 0 & 0 & 0 & 0 \\
\hline Cas 9 & 0 & 1 & 0 & 0 & 1 & 0 & 0 & 1 & 0 & 0 & 1 & 0 \\
\hline Cas 10 & 1 & 0 & 0 & 0 & 0 & 1 & 1 & 0 & 0 & 0 & 0 & 1 \\
\hline
\end{tabular}

Tableau 4. Analyse synthétique globale de l'appropriation de la FC

\begin{tabular}{|c|c|c|c|c|c|c|c|c|c|c|c|c|}
\hline \multicolumn{13}{|c|}{ Analyse synthétique globale de l'appropriation de la forêt communale } \\
\hline \multirow{3}{*}{ CAS } & \multicolumn{6}{|c|}{ PROPOSITION 1B } & \multicolumn{6}{|c|}{ PROPOSITION 2B } \\
\hline & \multicolumn{3}{|c|}{$\begin{array}{l}\text { COÛTS DE } \\
\text { TRANSACTIONS }\end{array}$} & \multicolumn{3}{|c|}{$\begin{array}{l}\text { MODES DE } \\
\text { GOUVERNANCE }\end{array}$} & \multicolumn{3}{|c|}{$\begin{array}{l}\text { SPECIFICITE } \\
\text { ACTIF }\end{array}$} & \multicolumn{3}{|c|}{$\begin{array}{l}\text { MODES DE } \\
\text { FINANCEMENT }\end{array}$} \\
\hline & CTB & CTI & CTE & REINT & SYNCO & GESRE & SAB & SAI & SAE & DET & DFP & FPE \\
\hline Cas 11 & 0 & 0 & 1 & 1 & 0 & 0 & 0 & 0 & 1 & 1 & 0 & 0 \\
\hline Cas 12 & 0 & 0 & 1 & 1 & 0 & 0 & 0 & 0 & 1 & 1 & 0 & 0 \\
\hline Cas 13 & 1 & 0 & 0 & 0 & 0 & 1 & 1 & 0 & 0 & 0 & 0 & 1 \\
\hline Cas 14 & 0 & 0 & 1 & 1 & 0 & 0 & 0 & 0 & 1 & 1 & 0 & 0 \\
\hline Cas 15 & 0 & 0 & 1 & 1 & 0 & 0 & 0 & 0 & 1 & 1 & 0 & 0 \\
\hline Cas 16 & 0 & 0 & 1 & 1 & 0 & 0 & 0 & 0 & 1 & 1 & 0 & 0 \\
\hline Cas 17 & 0 & 0 & 1 & 1 & 0 & 0 & 0 & 0 & 1 & 1 & 0 & 0 \\
\hline Cas 18 & 0 & 0 & 1 & 1 & 0 & 0 & 0 & 0 & 1 & 1 & 0 & 0 \\
\hline
\end{tabular}

CTB : coût de transaction faible; CTI : coût de transaction intermédiaire ; CTE : coût de transaction élevé.

1 : Structure de gouvernance efficace pour la Commune ; 0 : Structure de gouvernance non efficace pour la Commune.

SAB : spécificité actif bas ; SAI : spécificité actif intermédiaire ; SAE : spécificité actif élevé.

1 : Mode de financement efficient pour la Commune ; 0 : Mode de financement non efficient pour la Commune.

L'analyse synthétique des résultats du tableau 3 ci-dessus montre que sur les 18 cas étudiés, 10 cas concernent les transactions liées à l'appropriation de la terre et 8 concernent les transactions liées à 
l'appropriation de la forêt communale. Dans ce cas il s'agit des communes qui ont reçu de l'Etat le droit d'usage des parcelles de terres et des forêts dans leur territoire. S'agissant de l'appropriation des biens, la variable spécificité de l'actif, l'incertitude et la fréquence ont les coûts de transaction faible pour 8 cas soit $70 \%$ des communes. 2 communes (Cas 7 et 9 tableau 1 ci-dessus) sur 10 estiment qu'elles ont des coûts de transaction très élevés pour la Commune et pour le partenaire privé en ce qui concerne la spécificité des actifs, la solution serait le regroupement des communes donc les coûts de transactions pour l'appropriation des terres pour ces cas seraient intermédiaire et une commune (Cas 4 tableau 1 ci-dessus) sur 10 estiment leurs coûts de transaction sont élevés pour la Commune en ce qui concerne la spécificité des actifs, donc la régie intéressée serait adaptée. La gestion en régie représente donc la forme de gouvernance qui offre la solution la plus efficace pour ce qui est de l'appropriation de la terre, c'est-à-dire celle qui minimise le plus de coût. Par ailleurs, l'appropriation de la forêt communale indique 12,5\% des communes enquêtées soit 1 commune (cas 13 tableau 2 ci-dessus) sur 8 est capable de s'approprier l'exploitation de sa forêt communale. 12,5\% soit 1 commune (cas 16 tableau 2 en annexe) sont capable gérer sous forme de regroupement, les coûts de transactions pour l'appropriation des terres pour ces deux cas seraient intermédiaire. 6 communes soit 75\% exploitent leur forêt communale en régie intéressée, c'est-à-dire une forme de contrat commercial avec un exploitant forestier (partenaire privé). Pour l'appropriation de la forêt communale, la gestion en régie intéressée représente donc la forme de gouvernance qui offre la solution la plus efficace. La spécificité de l'actif étant l'attribut qui oriente le choix du mode financement. Nous constatons que l'estimation des coûts de transaction est faible en ce qui concerne l'exploitation de la terre et élevée pour ce qui est de l'exploitation de la forêt communale. Ainsi, le choix d'une structure de gouvernance et d'un mode de financement dépend du type de bien approprié et du degré de spécificité de l'actif. Cette structure financière transforme ainsi le développement économique, environnemental et social des Collectivités Territoriales. Les transactions des biens dans les communes camerounaises permettent de modifier à la fois la structure de gouvernance (gestion en régie, gestion en regie intéressée) et le mode de financement (fonds propres, fonds externes, fonds hybrides). Cette modification serait due en partie au degré de spécificité des actifs donc disposent ces Communes et de la nature bien au moment de la transaction. Ceci vient renforcer les études de [Williamson, 1985, 1991 ; Delmas, Ghertman et Obadia, 1997] sur le choix des structures de gouvernance et de [Cornell et Shapiro, 1987 ; Williamson, 1988 ; Vilasuo et Minkler, 2001] Sur le choix des modes de financement. L'analyse synthétique des résultats met en exergue les propositions empiriques suivantes :

P1A : La faible spécificité des actifs liée à l'appropriation des terres à un effet positif sur la gestion en régie dans les Communes Camerounaises

P2A : La faible spécificité des actifs liée à l'appropriation des terres nécessite un recours sur financement des fonds propres par les Communes Camerounaises

P1B : La forte spécificité des actifs liée à l'appropriation de la forêt communale ont un effet positif sur la gestion en régie intéressée dans les Communes Camerounaises.

P2B : La forte spécificité des actifs liée à l'appropriation de la forêt communale nécessite un recours sur financement des fonds externes par les Communes Camerounaises

\subsubsection{Discussion des résultats}

Les tableaux 3 et 4 ci-dessus font une analyse synthétique des formes de gouvernance et les modes de financement appropriés selon les principes qui ont été énoncés dans le rappel de la théorie. En particulier, les coûts de transaction ne sont pas calculés mais une estimation relative de chaque coût de transaction par mode de gouvernance, par mode de financement et par attribut est faite. Pour l'opportunisme les coûts de transaction dus à l'opportunisme des employés de la commune (gestion en régie) seront supérieurs à l'opportunisme dû au partenaire (gestion en régie intéressée) avec lequel la Commune aura fait un contrat de concession que ce soit l'appropriation des biens ou l'appropriation de la forêt communale. Comme l'indiquent les tableaux 1 et 2 ci-dessus, on peut estimer que les employés de la commune auront un niveau d'opportunisme supérieur à celui du sous-traitant, donc que les coûts de transaction dus à l'opportunisme seront plus élevés pour la commune que pour la solution de sous-traitance. Cet opportunisme s'explique par les négociations faites par les responsables communaux à tous les niveaux avec les partenaires privées, négociations visant a aboutir a des «contrats officieux» et à des «contrats officiels » avec pour finalité le versement régulier des «rentes obscures qui n'entrent pas dans les caisses de la commune (contrat officieux) » mais, plutôt dans les « comptes desdites responsables». Par contre, Si l'investissement en actifs 
est beaucoup plus important pour l'exploitation de la forêt communale, il n'en est pas de même pour la terre. Car dans la quasi-totalité des communes, le service technique est fonctionnel, et il existe au moins un carterpillar et un camion qui permet l'entretien des routes, tout au moins la commune pourrait supporter la location d'un camion pour réaliser les travaux de terrassement et d'ouverture des terres, propriétés de la Commune dans le but de transférer ces terres aux partenaires privés qui souhaitent investir dans l'industrie agricole, d'élevage, dans l'embourgeoisement rural ou bien dans la requalification. Le coût de l'actif spécifique sera plus bas dans la commune et élevé chez le partenaire privé, la solution serait la commune. Pourtant le coût de l'actif serait très élevé dans la commune en ce qui concerne l'appropriation de la forêt communale, car la gestion d'un titre de forêt à une durée de 30 ans et son exploitation nécessite des équipements (camions, carterpillar, GPS, Logiciels de gestion des forêts etc...). Il serait impossible pour une seule commune d'acquérir ou de louer des équipements adaptés pour l'exploitation d'une forêt. Car ces équipements pour la plupart sont estimés à des centaines de millions voire de milliards, "et lorsqu'on sait qu'il existe une dizaine de communes sur les 360 (excepté les mairies des villes) que compte le Cameroun a avoir un compte administratif dont le montant est compris entre un et quatre milliards, alors que nous nous n'atteignons même pas quatre cent millions, nous disait un receveur municipal » la solution serait alors automatiquement la gestion en régie intéressée car l'exploitant forestier dispose déjà tout cet équipement. Il est probable que le sous-traitant ne mettra pas assez de temps pour avoir ces actifs " même si nous n'avons pas les équipements sur place, nous pouvons les acquérir soit en achetant soit en louant du moment où les formalités contractuelles ont abouti, nous devrions faire des efforts car notre chiffre d'affaire peut en témoigner, nous rapportait un responsable technique d'une entreprise d'exploitation forestière » parceque les coûts de transaction dus à la spécificité des actifs sont plus élevés pour la Commune la solution serait la régie intéressée. Quant à l'incertitude, l'absence d'un organigramme type dans les communes qui prend en compte la gestion des terres et des forêts complexifie les travaux et les taches liées à l'exploitation desdits biens. Cette complexification est aussi due a des confusions dans les responsabilités, «tenez par exemple lorsqu'il y'a exploitation de la forêt c'est chaque employé qui se précipite pour aller donner ses orientations, négocier avec l'exploitant ou les services des eaux et forêts, tout le monde (Maire, Secrétaire général, Receveur Municipal, Sous-prefet, etc.....) nous confiait un responsable de la commune ». La législation externe favorise beaucoup l'Etat et les partenaires privés. Une rupture éventuelle du contrat ou le renouvellement du contrat à des conséquences importantes pour le partenaire privé que pour la Commune, car si les clauses contractuelles ne sont pas respectées, l'exploitant peut perdre son contrat, perdre son poste, sa notoriété ainsi que celle de son entreprise. Le Maire est un élu du peuple dont il est difficile de le faire partir, même en cas de non atteinte des objectifs. Les coûts de transactions liés à l'attribut d'incertitude seront plus élevés pour le partenaire privé que la dans la commune. Concernant la fréquence des transactions, les coûts de transaction sont faibles pour la Commune, puisqu'ils se résument au moment des élections, alors que les coûts de transaction liés au contrat de sous-traitance devront normalement être renouvelés plus fréquemment. En plus, les différentes démarches d'obtention des permis (permis de construire et permis annuel des opérations) devront être effectués régulièrement. Les coûts de transaction seront plus importants pour le partenaire privé que pour la Commune. La solution serait donc la Commune. Les coûts de transaction dus à l'appareil bureaucratique seront plus élevés pour la Commune que pour le partenaire privé puisque, la commune est régie par des conventions, donc soumises à de nombreux contrôle de la part de la tutelle technique et financière. L'objectif est de veiller aux procédures édictées par l'Etat. Des rapports et un contrôle existent également dans la sous-traitance mais ils sont par nature moins élevés. Dans le cas de la bureaucratie, la gestion par la régie intéressée comme mode de gouvernance est privilégiée. Pour les incitations par contre, le responsable du partenaire privé aura une incitation très forte à remplir ses objectifs puisque l'avenir de sa carrière et de l'entreprise en dépend, alors que le Maire de la Commune fera passer ses objectifs individuels avant ceux de ses citoyens. La solution serait alors d'opter pour la gestion en régie. Les coûts liés aux incitations seront faibles pour la commune que le partenaire privé, la solution la plus efficace serait la Commune. «Nous ne savons qui est-ce que nous pouvons confier tel ou tel tache, nous n'avons pas les employés qui sont spécialisés dans la foresterie ou dans les travaux de bornage des terrains ou tout autre travaux allant dans le transfert des terres aux particuliers. La Commune ne dispose pas assez de ressources financières pour recruter des spécialistes dans tel ou tel domaine, nous a confié un Secrétaire général de Mairie », Les coûts de transactions dus aux compétences sont élevés pour la commune que pour le partenaire privé car ce dernier dispose déjà des ressources humaines qualifiés et des moyens nécessaires pour les rémunérer, la solution serait alors d'opter pour la régie intéressée. Les tableaux 3 et 4 indiquent des 
coûts de transaction variables (1,2 ou 3 et 4 cf tableaux 1 et 2 ci-dessus) selon l'attribut des modes de gouvernance. Il faut donc trouver un moyen simple de voir quel est le mode de gouvernance le plus efficace, c'est-à-dire celui qui économise le plus des coûts de transaction, mais aussi le mode de financement le plus efficient. Dans le cas de l'appropriation des deux biens, on remarque qu'opportunisme, bureaucratie et compétences ont des coûts de transaction plus bas pour le partenaire privé alors que l'attribut incitations à des coûts de transaction plus faibles pour la Commune. Même s'il est difficile d'estimer le poids relatif de chacun de ces quatre attributs dans le total des coûts de transaction, on peut penser que le résultat différentiel net de ces quatre variables va être très faible quelque soit le bien approprié. Cela permet donc de ne plus les compter pour faire le choix. Par contre, concernant les trois autres attributs des transactions : spécificité des actifs, incertitude et fréquence, la Commune représente la solution la plus efficace pour l'appropriation de la terre et moins efficace pour l'appropriation de la forêt communale, car les coûts liés à l'attribut spécificité de l'actif sont très élevé. Bien que les coûts liés aux attributs incertitude et fréquence soient faibles pour la Commune et élevés pour le partenaire privé, le poids relatif aux actifs spécifiques que sont des équipements, les camions et les caterpillars..... reste l'attribut déterminant de la structure de gouvernance dans les communes. En nous appuyant aussi sur les réponses de nos interviewés, le coût des équipements liés à l'exploitation de la forêt communale reste un attribut qui défavorise la gestion en régie par les communes. Il est facile de conclure, dans ce cas, que les coûts de transaction dus au mode de gouvernance-Commune seront globalement inférieurs à ceux dus aux mode de gouvernance-partenaire privé pour la terre et qu'il faut donc choisir la gestion en régie, en outre l'exploitation du foncier nécessite des actifs spécifique bas d'où un recours au financement propre. Par contre les coûts de transaction dus au mode de gouvernance-Commune seront globalement supérieurs à ceux dus aux modes de gouvernance-partenaire privé pour l'exploitation de la forêt communale, dans ce cas la gestion régie intéressée est appropriée, la commune doit faire recours au financement externe pour réaliser les travaux parceque les actifs spécifiques sont très élevés. On voit donc bien que le cadre de l'analyse discrète des modes de gouvernance proposé par [Williamson, 1991] et simplifié dans les tableaux 1 et 2 (ci-dessus) tient compte de la nature bien concernée. Nous avons comparé la solution de la Commune, à celle du partenaire privé et du regroupement des communes et avons bien vu que le mode de gouvernance choisi varie en fonction du dégré de spécificité des actifs et du bien approprié. Mais le mode de financement choisi dépend du vocable spécificité des actifs incluant l'incertitude, la fréquence de la transaction du bien approprié.

\subsection{Principaux apports de l'étude}

L'étude à pour objectif de comprendre l'effet de l'appropriation des biens sur la structure des Communes Camerounaises. Cet effet est lié à leur développement économique, environnemental et social.

Du point de vue du développement économique, la structure gestion efficace contribue à réduire les coûts de mesure et de contrôle de l'activité économique [Barzel, 1982] en vue de garantir l'amélioration des recettes locales pour un meilleur traitement des employés et des parties prenantes (les citoyens, les riverains, les contribuables, les fournisseurs, etc...). Cette situation permet d'atténuer des conflits et de bonifier l'autonomie financière (valeur) à long terme des Communes. Divers théories expliquent cette approche, la théorie des contrats implicites de travail [Holmstrôm, 1983 ; Drèze et Gollier, 1993] avancent l'argument qu'en offrant une assurance implicite aux travailleurs risquophobes contre les fluctuations de leur productivité en valeur, l'employeur peut dégager un revenu supplémentaire en provenance de cette activité d'assurance. La théorie du salaire d'efficience, [Krueger et Summers, 1988] explique qu'une entreprise qui offre des salaires supérieurs à ses concurrents lui permet de mieux motiver ses employés et d'attirer les meilleurs candidats sur le marché, ce qui peut avoir un effet net positif sur sa performance financière. [Williamson, 1994, p. 111] s'intéresse aux arrangements institutionnels beaucoup plus adapté à la réalité du monde des contrats. Des lors le problème est de savoir le type de contrat qui doit être mise en place pour minimiser les coûts de transaction. La théorie des coûts de transactions permet donc de garantir les recettes locales dans les Communes en choisissant soit la hiérarchie qui est ici la gestion en régie [Williamson 1975, 1985] utilise la hiérarchie pour résoudre les problèmes de spécificité des actifs et d'opportunisme et [Barzel, 1982] pour réduire les coûts de mesure et de contrôle de l'activité économique, soit la forme hybride qui est la régie intéressée, selon la nature du bien et la spécificité des actifs. La théorie de l'agence [Jensen et Meckling, 1976] va insérer des mécanismes incitatifs (responsabilisation des acteurs locaux, développement des infrastructures...) dans des contrats pour harmoniser les intérêts des agents et ceux des détenteurs de capital dans le but de réduire le problème d'asymétrie informationnelle conduisant à une sélection adverse et 
à des comportements opportunistes des responsables municipaux. Ceci signifie que la structure des communes peut être le résultat d'un calcul traditionnel de maximisation des recettes. Dans un tel contexte, la logique collective de l'appropriation des biens aurait un impact sur l'allocation des ressources dans l'économie et sur le bien-être des parties prenantes. La logique privée de l'appropriation des biens elle serait plutôt une stratégie opportuniste des investisseurs attirés par des communes toutes aussi opportunistes pour la logique collective.

Du point de vue du développement environnemental et social, la structure contribue à la prise en compte des externalités et le développement durable. Le problème d'externalité décrié depuis longtemps par les économistes est l'une des sources les plus abondantes d'inefficacité des marchés. L'exploitant forestier ne tient pas compte de l'effet de la coupe sur la disponibilité de la ressource pour les générations futures, et ne se sent pas concernée par l'utilisation injuste des royalties perçues par les élites locale et le gouvernement corrompu. L'existence d'externalités introduit une divergence entre profit privé et bénéfice social. Parce qu'il n'internalise pas les dommages qu'il cause à autrui, l'entrepreneur-pollueur polluera plus qu'il n'est socialement désirable. La structure financière permet de réduire ces externalités en introduisant une approche alternative qui consiste a donner un prix aux externalités produites et à le faire payer par les producteurs de ses externalités, à charge pour eux de décider de modifier leur mode de production pour éviter de devoir payer ce prix. C'est le principe " pollueur-payeur » appliqué à l'ensemble des problèmes d'externalité. Il peut être mis en œuvre par une taxe, ou par un système de marché de permis d'émission. Si le niveau du prix correspond à la valeur monétaire des impacts de ces externalités sur le bien-être des parties prenantes, le producteur de l'externalité est en situation d'internaliser les conséquences de ses décisions sur l'ensemble de la société. La logique d'appropriation des biens vient résoudre la divergence entre bénéfice privé et bénéfice social, en confortant le principe «polleur-payeur» pour la logique privée et l'accroissement de l'« épargne de précaution » à des générations futures pour la logique collective. Ce qui suppose de faire un sacrifice courant afin de rendre l'environnement institutionnel crédible dans le but d'intensifier les transactions entre agents économiques pour promouvoir l'investissement local, car les incitations dans ce cadre sont positives [North, 1981, 1990, 1994]. Cependant, les économistes recommandent que l'évaluation d'actes collectifs aux conséquences incertaines fasse l'objet d'une évaluation socio-économique basée sur son impact et sur l'espérance d'utilité des parties prenantes, où l'utilité de celles-ci est une fonction concave de leurs consommations. Ceci permet de déterminer un impact " équivalent certain », c'est-dire l'accroissement de consommation certaine qui aurait le même effet sur le bien-être des parties prenantes que le risque qu'on leur impose effectivement. Dans le cadre de la logique de l'appropriation des biens, les retombées financières et infrastructurelles que les populations perçoivent de la logique privée auraient le même effet sur leur bien être en termes d'érosion de la culture, de destruction des champs villageois, d'effets pervers sur la population (grossesses indésirées des jeunes filles, charge supplémentaires des parents des jeunes filles, divorce régulier auprès des couples), diverses maladies contractées par les populations locales (cas ou le partenaire privé va exploiter la foret communale avec toute son équipe). Ou bien la compensation financière de certains organismes de financement lorsque la Commune choisit la conservation de la forêt serait l'équivalent que la commune aurait bénéficiée si elle avait choisi la logique collective. Par ailleurs pour ce qui est de l'appropriation de la terre propriété de la Commune, les impôts liés à la reconversion d'une élite agricole, à l'embourgeoisement rural et aux requalifications perçues par la Collectivité aurait le même effet sur les bénéfices que la Commune engendrerait à moyen et à long terme. La structure de financière prend en compte l'impact équivalent certain dans l'analyse coût-bénéfice en situation de transaction des biens entre agents économiques.

\section{Conclusion}

La logique transactionnelle de l'appropriation des biens dans les Communes Camerounaises avait pour objectif de comprendre comment l'appropriation des biens contribue à la transformation du développement local. Les Communes disposant des droits d'usage, de jouissance et de disposer des biens à travers à travers la loi N²019/024 du 24 Décembre 2019 portant code général des Collectivités Territoriales Décentralisées procèdent régulièrement à de nombreuses transactions vers des partenaires privées ou bien en jouissent ellesmêmes. Ces transactions sont liés à des attributs que sont l'opportunisme, la spécificité des actifs, l'incertitude, la fréquence, l'incitation, la bureaucratie et les compétences quelque soit la logique de transactionnelle, privée ou collective. Il s'en suit que le vocable spécificité de l'actif transforme la structure de gouvernance et le mode de financement dans les Communes selon les deux biens étudiés, la terre et la 
forêt communale. Pour ce qui est de l'appropriation de la terre, la gestion en régie est la structure de gouvernance efficace, car elle minimise les coûts de transaction. La spécificité des actifs n'étant pas élevée, la commune devrait opter pour un mode de financement par fonds propres pour l'exploitation de ces terres. S'agissant de l'appropriation de la forêt communale, la gestion en régie intéressée est la structure de gouvernance efficace, car les coûts de transaction de la commune sont très elevés. La spécificité des actifs est élevée, la commune devrait opter pour un mode de financement par emprunt externe pour l'exploitation de sa forêt communale. Cet emprunt devrait lui permettre d'acquérir les investissements et les équipements nécessaires pour exploiter elle-même sa propre forêt communale, au cas contraire elle devra céder l'exploitation à un partenaire privé. La logique d'appropriation (collective ou privée) dépend ainsi de la spécificité des actifs et de la nature du bien à s'approprié par la Commune. La transformation de la structure dans les communes à donc un impact considérable sur le développement local. La modification de la structure accroit les recettes communale, réduit les conflits avec les employés, les conseillers municipaux et les autres parties prenantes, réduit les externalités en introduisant le principe « pollueur-payeur » appliqué à l'ensemble des problèmes d'externalité enfin elle tranche l'indécidable en situation d'incertitude a travers le principe de 1' "équivalent-certain » basé sur l'espérance d'utilité des parties prenantes à l'appropriation des biens. Les structures de gouvernance et financière sont un régulateur du développement local. Elles permettent donc de choisir un support institutionnel efficace et un mode de financement efficient pour le plan communal de développement. Ce régulateur promeut ainsi les principes de gouvernance territoriale, de participation citoyenne des acteurs locaux et de l'entrepreneuriat local. Les Communes des pays Camerounaises peuvent dès lors promouvoir l'intercommunalité ou le regroupement des communes pour une logique collective d'appropriation des biens soutenu à moyen et à long terme.

\section{Bibliographie}

1. ABIMBOLA A. (2002), Cross-sectional test of pecking order hypothesis against static trade-off théory on UK data, Working paper.

2. AGRAWAL A. AND E OSTRO. (2001), «Collective action, propriety rights and decentralization in resource use in India and Nepal », Politics and Society, Vol. 29, $\mathrm{N}^{\circ} 4$. pp. 485-514.

3. AGRAWAL A. AND J. C. RIBOT. (1999), « Accountability in decentralization: A Framework with South Asian and West African cases », The Journal of Developing Areas, 33 (summer), pp. 473502.

4. ALCHIAN A. AND H DEMZETZ. (1972), " Production, Information Cost and Econmic Organization » American Econmic Review 62 (December): 777.795.

5. ANDERSON J. ET AL. (2006), «Forests, poverty and equity in africa: new perspectives on policy and practice», International Forestry Review, vol. 8, n 1 , pp. 44-53.

6. BARTON S L. AND GORDON P J. (1987), «Corporate Stratégy : Useful Perspective For the Study of Capital Structure », Academy of Management Review, pp. 67-75.

7. BAEZEL Y. (1982), «Measurment Cost and the Organization of Markets», Journal of Law and Economics, 25: 27-48.

8. BISSUEL, R. (2010). Etude sur le potential de developpement des zones industrielles en mutation dans une perspective de requalification urbaine orrientée sur les transports en commun, memoire de maitrise, Université du Quebec à Montréal, Département d'etudes Urbaines.

9. BLAIR H. (2000), «Particpation and accountability at the périphery : démocratic local governance in six countries », World developpement, Vol. $28 \mathrm{n}^{\circ} 1$, PP. . 21-39.

10. BOOTH L. AIVASIAN V. DÉMIRGUC-KUNT A. MAKSIMOVIC V. (2001), «Capital structure in developing countries », Journal of finance 56, pp. 87-130.

11. CHAKRABORTY I. (2010), «Capital structure in an emerging stock market: the case of India». Research in International Business and Finance 24, pp. 295-314.

12. CHENG S R. SHIU C Y. (2007). «Investor protection and capital structure : International evidence», Journal of Multinational Financial Management 17, pp. 30-44.

13. CORNNEL B. SHAPIRO A. (1987). «Corprate Stakeholders and corporate finance», Financial Management, pp. 5-14.

14. DELMAS, M. (1996), Stratégie d'Acquisition Technologique dans le Secteur du Traitement des déchets Spéciaux en Europe et au Etats Unis, Thèse de Doctorat, Groupe HEC.

15. DELMAS M. GHERTMAN M. AND HIEMAN B. (1997), Institutional Environment Effects on 
Transaction Costs : a Comparative Analysis of the US and French Nuclear Power Industries, in Thomas, O'Neal and Ghertman, Stratégy, Structure and Style, Wiley.

16. DREZE J H. AND C GOLLIER. (1993). «Risk Sharing on the Labour Market and Second-Best Wage Rigidity», Européan Econmic Review, 37, 1457-1482.

17. ELLSBERG D. (1961). «Risk, ambiguity, and the Savage axioms», Quaterly Journal of Economics, 75, pp 643-690.

18. GERTHMAN M. (2003), «Oliver williamson et la théorie des couts de transactions », Revue Française de Gestion, ${ }^{\circ} 142$, pp 43-63.

19. GIASSON I. M PROVOST. (2000). «Essai de réqualification d'un site à contrainte environnementales : le complexe environnemental Saint-Michel, dans: Gilles sénechal et Diane saint-laurent (dir), les espaces dégradés »: contraintes et conquetes, Presses de l'Université du Quebec, p. 187-189.

20. GOMEZ P Y. (1996), Le gouvernement des entreprises, Economica, Paris

21. GUIMOND L. ET M SIMARD. (2005) «Néo-ruralité et embourgeoisement des Campagnes quebécoises: un regard nuance, »Actes du XLVè Colloque international de l'Association de Sciences Regionale de langue francaises (ASRDLF) Térritoires et action publique territorial: nouvelles ressources pour le développement regional, Rimouski, 25-27 aout 2008, Université du Québec à Rimouski (UQAR).

22. HOLMSTRON, B. (1983), «Equilibrium Long-Term Labor Contracts», Quaterly Journal of Economics, Supplément, 1983.

23. HUANG G. SONG F. M. (2006) «The determinants of capital structure : Evidence from china», China Econmics Review 17 (1) pp. 14-36.

24. HUMEL R. AND A SIZYKH. (1997) «Sustainaible developpement of forests as a way to preserve the natural basis of forestry», Journal of Sustainable Forestry, vol. 4, n³/4, pp. 53-60.

25. JENSEN M C ET MECKLING W (1976), «Théory of de firm : Managérial Behavior, Agency Cost and Ownership Structure », Journal of Financial Economics, vol. 3, pp. 305-360.

26. JÉREMIE M. (2009), «De la decentralisation de la gestion forestière à une gouvernance locale des forêts communautaires et des rédevance forestières au Sud-est Cameroun», VertigO - la revue electronique en sciences de l'environnement, Volume 9, numéro 1.

27. KRUEGER AND SUMMERS. (1988), «Efficiency Wages and the Inter-Industry Wage Structure», Economtrica, 56 (2), Mar 1988, 259-293.

28. MASTEN, S. (1996), Empirical Research in transaction Cost Economics: Challenges, Progress, Direction, in G. Groenwegen (ed), Transaction Cost Econmics and Beyond, Boston, Kluwer Académic Publishers.

29. MAUREL M C. (1994), La transition post-collectiviste. Mutations agraires en Europe central. L'Harmattan, $360 \mathrm{p}$.

30. MAUREL M C. (2012), «Stratégies d'appropriation de la terre et du capital dans les agricultures centre-européennes», Cahiers du CEFRES, Centre Francais de Recherche en Sciences Sociales, Anciens et nouveaux propriètaires (ed Anne Olivier).

31. MYERS S C. ET N MAJLUF. (1984) «Corporate Financing and investment decisions when Firms have information that investors do not have », Journal of Financial Economics, vol. 13, pp. 187-224.

32. NORTH D. (1981), Structure and Change in Economic History, W.W. Norton, N.Y.

33. NORTH D. (1990), Institutions, Institutionnal Change, and Economic Performance, Cambridge University Press, New York.

34. NORTH D. (1994), «Economic Performance through Times», The Américan Economic Review, vol, $84, n^{\circ} 3$, p. 359-368.

35. PERRENOUD. (2008), «Les artisans de la gentrification rurale: trios manières d'etre macons dans les Hautes-Corbières», Sociètés contemporaines, 3, 71: 95-115.

36. PROST B. (1991), «Du rural au péri-urbain: conflit de territoire et requalification de l'espace», Revue de géographie de Lyon, 2 , 66:96-102.

37. RAJAN R G. ZINGALÈS L. (1995), «What do we Know about capital structure? Some Evidence from international Data, Journal of Finance, 50 (5), pp. 1421-1460.

38. RIBOT J. C. (2003), «Democratic decentralization of natural resources: Institutionnal Choice and discretionary power transfers in Sub - Saharan Africa», Public administration and development, 
vol. $23 \mathrm{n}^{\circ} 1$, pp. 1-18.

39. RIPOLL ET V VESCHAMBRE. (2005), L'appropriation de l'espace comme problématique, Norois, 195: 1-10.

40. SIMARD M. (2007), «Nouvelles populations rurales et conflits au Quebec: regards croisés avec la France et le Royaume-Uni», Geographie, economie, socièté, 9: 187-213.

41. SIMARD M. ET L GUIMOND. (2012). «Que penser de 1'embourgeoisement rural au Quebec? Visions différenciees des acteurs locaux», Recherches sociographiques, 53, 3: 527-533.

42. SIMON H. (1945). Administrative Behavior, The Free Press

43. STEINER S. (2007). «Decentralisation and poverty: conceptual framework and application to Uganda», Public administration and development, vol. 27, pp. 175-185.

44. VILASUO J. MINKLER A. (2001), «Agency costs, asset specificity, and the capital structure of the firm», Journal of Economics Behavior and Organization 44, pp. 55-69.

45. Yin R.K. (1994), Case study research: Design and methods (2nd ed.). Beverly Hills, CA: Sage Publishing.

46. WILLIAMSOM O E. (1975). Markets and Hierarchies: Analysis and Antitrust Implications. New York: The Free Press.

47. WILLIAMSOM O E. (1985), The Economic Institutions of Capitalism. New York: The Free Press.

48. WILLIAMSOM O E. (1988), «Corporate Finance and Corporate Governance», Journal of Finance 43 (3), pp. 567-591.

49. WILLIAMSOM O E. (1991), «Comparative Economic Organization: The Analysis of Discrète Structural Alternative». Administrative Science Quaterly 36: 269-296.

50. WILLIAMSON O E. (1994), Les institutions de l'economie, traduit de : The Economics Institutions of Capitalism (1985), InterEditions, Paris. 